\title{
GV20 Penetrating GB7 on the Expression of Wnt1 in Brain Tissue of Rats with Intracerebral Hemorrhage
}

Wei Wu ${ }^{1}$, Xiaohong Dai ${ }^{2}$, Wei Zou ${ }^{2,3 *}$, Xueping Yu${ }^{2 *}$, Wei Teng ${ }^{2}$, Xiaowei Sun ${ }^{2}$, Weiwei Yu ${ }^{2}$, Huihui Ma ${ }^{2}$, Qiuxin Chen ${ }^{2}$, Peng Zhang ${ }^{1}$, Tingting Yu and Peng Liu ${ }^{1}$

${ }^{1}$ Heilongjiang University of Chinese Medicine, 24 Heping Road, Xiangfang District, Harbin, Heilongjiang, 150040, China

${ }^{2}$ First Affiliated Hospital of Heilongjiang University of Chinese Medicine, 26 Heping Road, Xiangfang District, Harbin, Heilongjiang, 150040, China

${ }^{3}$ Key Laboratory of Heilongjiang University of Chinese Medicine, 24 Heping Road, Xiangfang District, Harbin, Heilongjiang, 150040, China

\begin{abstract}
Background: Intracerebral hemorrhage $(\mathrm{ICH})$ is a life-threatening disease that confuses us for centuries. Acupuncture as a kind of Chinese traditional treatment has been empirically established and widely used in clinics in China, especially for stroke victims. Studies proved that GV20 penetrating GB7, a kind of needling manipulation, with which a needle is inserted into GV20 and then to GB7 under the scalp, is especially effective on the treatment of this disease. Wingless-type1 (Wnt1) is a key factor of Wnt/ß-catenin signal pathway, which could promote regeneration and remodeling of neural function after $\mathrm{ICH}$ by affecting proliferation and differentiation of the neural stem cells (NSCs). The study on the expression of Wnt1 induced by GV20 penetrating GB7 may give out the theoretical principle of the treatment.
\end{abstract}

Objective: The goal of this work is to observe the effect of GV20 penetrating GB7 needling on the protein/ mRNA expression of Wnt1 in brain tissue of $\mathrm{ICH}$ rats.

Methods: In this experiment, we focused on observing the protein/mRNA expression level of Wnt 1 by Western blot and real-time fluorescent quantitative PCR (RTFQ PCR) in the rats with ICH, which were treated by Dickkopfrelated protein 1 (DKK1), acupuncture or no intervention, comparing with the healthy rats without any treatment at fixed time points.

Results: This study revealed that the expression of Wnt1 had the similar trend in all groups at each time point of corresponding treatment, which began to increase from the third day (3d), reached the peak on the seventh day (7d) then decreased gradually. The acupuncture treatment group showed a higher level of expression.

Conclusion: The results show that GV20 penetrating GB7 has advantage on promoting the protein/mRNA expression of Wnt1, activates the intracellular Wnt pathway, and regulates the transcription of downstream target genes, affecting proliferation and differentiation of the neural stem cells (NSCs), thereby initiates endogenous repair mechanisms of the organism itself. This study provides a powerful scientific basis for acupuncture treatment on $\mathrm{ICH}$.

Keywords: GV20 penetrating GB7; Wnt1; Intracerebral hemorrhage; Neural stem cells

\section{Introduction}

Intracerebral hemorrhage $(\mathrm{ICH})$ is a non-traumatic parenchymal hemorrhage [1]. Most of the patients suffer from hemiplegia or cognitive impairment because of the absence of a large number of neurons, which is difficult to be cured by the current treatment. Studies in recent years found that a variety of adult mammals have proliferation ability of neural stem cells (NSCs) in the sub ventricular zone (SVZ) and the sub granular zone (SGZ) [2] of dentate gyrus of hippocampus. Ischemia, hypoxia, injury and other pathological stimuli can induce the proliferation and differentiation of endogenous NSCs in SVZ and SGZ $[3,4]$. The $\mathrm{Wnt} / \beta$-catenin signaling pathway plays an important role in the development of the mammals central nervous system and the regulation of proliferation and differentiation of NSCs. ICH stimulates cell proliferation and migration from the SVZ to the injured tissue $[5,6]$. The use of endogenous regeneration mechanisms in the treatment of stroke patients after ICH seems to be a rational idea and the mechanism of this treatment needs to be explored deeply.

Scalp acupuncture treatment of ICH began to be applied in our hospital from the early 1990s, and a large number of basic studies have been carried out since then. From 2007 to 2009, we employed GV20 penetrating GB7 needling method and treated 144 patients that were randomly divided into control group and acupuncture group. The recovery rate was $58.33 \%$, and the total effective rate was $91.67 \%$ [7]. Our previous research on GV20 penetrating GB7 showed that acupuncture can activate endogenous NSCs by regulating external signal, such as neurotrophic factors [8]. Based on this research, we prepared rat model of ICH and detected dynamic changes of the protein/mRNA expression of Wnt1 by real-time fluorescent quantitative PCR (RTFQ PCR) and Western blot, with Dickkopf-related protein 1 (DKK1) as the antagonist of Wnt signaling pathway. The next step is to comparatively observe the influence of GV20 penetrating GB7 on the expression of Wnt1 in brain tissue of ICH rats in order to explore the overall regulating action of the intracellular signal transduction pathway and clarify the mechanism of the acupuncture treatment in promoting

*Corresponding authors: Wei Zou, Key Laboratory of Heilongjiang University of Chinese Medicine, 24 Heping Road, Xiangfang District, Harbin, Heilongjiang, 150040, China, Tel: +8645182161746; E-mail: kuangzou1965@163.com

Xueping Yu, First Affiliated Hospital of Heilongjiang University of Chinese Medicine, 26 Heping Road, Xiangfang District, Harbin, Heilongjiang, 150040, China, Tel: +8645182161746; E-mail: hzyyxp82@163.com

Received December 08, 2017; Accepted December 13, 2017; Published December 19, 2017

Citation: Wu W, Dai X, Zou W, Yu X, Teng W, et al. (2017) GV20 Penetrating GB7 on the Expression of Wnt1 in Brain Tissue of Rats with Intracerebral Hemorrhage. Med Chem (Los Angeles) 7: 388-392. doi: 10.4172/2161-0444.10485

Copyright: (c) $2017 \mathrm{Wu} \mathrm{W}$, et al. This is an open-access article distributed under the terms of the Creative Commons Attribution License, which permits unrestricted use, distribution, and reproduction in any medium, provided the original author and source are credited. 
neural remodeling after ICH. Thus, this study provides a new scientific basis and theoretical principle for the acupuncture treatment of ICH.

\section{Materials and Methods}

\section{Chemistry}

All the reagent and instruments in this experiment were purchased locally and were of analytical grade. Acupuncture needles of Hwato, $0.35 \times 40 \mathrm{~mm}$ were used in the acupuncture treatment of GV20 penetrating GB7. The experimental rats were fixed in prone position on the stereotaxic instrument (Chengdu Instrument Factory, STW-1). Engine bit (Shanghai dental Machinery Factory, 307-6) was used to drill a hole in each skull of the rat. Primary antibodies (Rabbit-anti Wnt1, Beijing Boosen), which bind to non-antibody antigen, and secondary antibodies (Goat-anti-Rabbit, Beijing Boosen), which bind to antibody, were both applied in Western blot. GEL imaging system (Alpha Innotech, USA) was employed to detect and analyze different staining through non chemiluminescence imaging of gel electrophoresis (protein/mRNA). Nucleic acid amplification instrument (ABI9700, USA) was supplied for RTFQ PCR, and vertical electrophoresis transfer membrane system (Bio Rad, USA) was used for electrophoresis and transferring membrane.

\section{Animals}

Adult male Sprague-Dawley (SD) rats with average weight of 250 $\mathrm{g} \pm 20 \mathrm{~g}$ were used in the experiment. The animals had free access to food and water. The experiment was performed in accordance with the current guidelines for the care of laboratory animals and the ethical guidelines for the investigation of experimental pain in conscious animals.

\section{Sample collection and processing}

ICH molding: 192 healthy male SD rats were chosen randomly, 12 were assigned to blank group and 180 were made to ICH models. Firstly the rats were fixed on the stereotaxic instrument in prone position after being anesthetized and the scalp skin being shaved. Sagittal incision was made in the scalp to expose the anterior fontanel. The micro syringe was fixed with stereotaxic instrument and aimed at anterior fontanel, then was moved $3.5 \mathrm{~mm}$ to the right side and 0.2 $\mathrm{mm}$ to the back. A sign was marked here, and a hole was drilled in the skull with engine bit on the sign. This is the basal ganglia area of the rat brain. Meanwhile, $60 \mu$ autologous blood was collected from the rat tail with microinjector and slowly injected the blood into caudate nucleus $6 \mathrm{~mm}$ beneath the surface of the brain through the hole. The needle was withdrawn after 3-5 min. The hole was closed with dental cement, sterilized with gentamicin, and sutured.

Neurological score: Berdersonscore was used to evaluate the neurological function of the rats. Based on this scoring, rats were held gently by the tail, and suspended one meter above the floor; normal rats extend both forelimbs towards the floor (Table 1) [9]. After the ICH molding, we counted the rats with 1-3 grade as successful ones, which were included in the experiment.

Intervention: The 180 rats to be made into ICH models were first randomized into 3 groups, and 60 rats were in each of them. $10 \mu \mathrm{L}(0.1$

\begin{tabular}{|c|c|c|}
\hline Normal & Grade 0 & No observable deficit \\
\hline Moderate & Grade 1 & Forelimb flexion \\
\hline \multirow{2}{*}{ Severe } & Grade 2 & $\begin{array}{r}\text { Decreased resistance to lateral push (and forelimb } \\
\text { flexion) without circling }\end{array}$ \\
\cline { 2 - 3 } & Grade 3 & Same behaviour as Grade 2, with circling \\
\hline \multicolumn{3}{|c|}{ Table 1: Neurological Examination Grading System. }
\end{tabular}

$\mu \mathrm{g} / \mu \mathrm{l}) \mathrm{GSK}-3 \beta$ inhibitor DKK1 was injected into the rats in DKK1 group 30 min before modeling, GV20 penetrating GB7 needling was applied to the rats in acupuncture group after modeling, and no intervention was employed to the rats in model group either before or after molding. Furthermore, each group was divided into five subgroups by the time point: the third day subgroup (3d), the seventh day subgroup (7d), the fourteenth day subgroup (14d), the twenty-first day subgroup (21d) and the twenty-eighth day subgroup (28d). GV20 penetrating GB7 needling was applied to the rats in acupuncture group for $30 \mathrm{~min}$ each time, once a day. 5 min twisting manipulation was given once in each $10 \mathrm{~min}$ at the speed of $200 \mathrm{rpm}$. We sampled the three groups of rats above at corresponding time point. The blank group of rats received no intervention and were sampled directly. All animals were sacrificed after the samples were taken. Out of every twelve samples in each group/subgroup, six were detected for the expression of Wntl by Western blot and the other six by RTFQ PCR.

Western blotting: SDS-PAGE gel was prepared first. The samples were boiled for $5 \mathrm{~min}$, placed on ice and centrifuged for 10 minutes at $4^{\circ} \mathrm{C}$ under $12000 \mathrm{rpm}$ after the sample tissues were ground, and protein lysate was added. Then the supernatant was dropped into the centrifuge tube. The protein in the sample was measured, and then some sample was taken with injector for electrophoresis, transferring membrane and blocking. Appropriate concentration of primary antibodies was added into the blocking solution. The mixture was kept overnight at $4^{\circ} \mathrm{C}$ and rewarmed on the following day. Then appropriate concentration of secondary antibodies was added in the sample.

RTFQ PCR: PCR buffer, dNTP, primers and DNA template, etc. were added separately in a centrifugal tube on ice. The mixture was centrifuged, amplified in PCR amplification instrument, and detected through PCR electrophoresis.

\section{Statistical analysis}

Data were analyzed with the help of a SPSS version 22 , and the results were expressed as Mean \pm Standard Deviation (SD). Comparison between groups was performed with one-way analysis of variance (ANOVA). $\mathrm{P}<0.05$ was considered to be statistical significance.

\section{Results}

Protein expression of Wnt1 in the brain tissues of each group by Western blot

The Western blot results showed that the protein expression is in extremely low level in the blank group. The protein expression of Wnt1 in the model group began to increase from $3 \mathrm{~d}$, reached the peak at $7 \mathrm{~d}$ then decreased gradually in $14 \mathrm{~d}, 21 \mathrm{~d}$, and $28 \mathrm{~d}$. The protein expression of the model group at each time point were statistically significant compared with the blank group $(\mathrm{P}<0.05)$. The acupuncture group at each time point expressed the same trend with the model group, but the protein expression in $7 \mathrm{~d}, 14 \mathrm{~d}, 21 \mathrm{~d}, 28 \mathrm{~d}$ was significantly higher than that of the model group at the same time points. The results were statistically significant $(\mathrm{P}<0.05)$. DKK1 group had the same expression tendency with the model group at each time point, but the protein expression significantly decreased than that of the same time point of the model group in $7 \mathrm{~d}, 14 \mathrm{~d}, 21 \mathrm{~d}, 28 \mathrm{~d}$, and compared with the model group and the acupuncture group at the same time point, the results were statistically significant $(\mathrm{P}<0.05)$ (Figures 1 and 2$)$.

\section{mRNA expression of Wnt1 in the brain tissues of each group by RTFQ PCR}

The RTFQ PCR results showed that mRNA expression was at the 
lowest level in the blank group. The level of mRNA expression of Wnt1 in the model group was slightly higher in $3 \mathrm{~d}$, reached the peak at $7 \mathrm{~d}$ then decreased gradually in $14 \mathrm{~d}, 21 \mathrm{~d}$ and 28 . The mRNA expression of the model group at each time point were statistically significant compared with the blank group $(\mathrm{P}<0.05)$. The acupuncture group at each time point expressed the same trend with the modle group, but the mRNA expression in $7 \mathrm{~d}, 14 \mathrm{~d}, 21 \mathrm{~d}, 28 \mathrm{~d}$ was significantly higher than the model group at the same time point. The results were statistically significant $(\mathrm{P}<0.05)$. DKK1 group had the same expression tendency with the model group at each time point, but the mRNA expression significantly decreased than that of the same time point of the model group in $7 \mathrm{~d}, 14 \mathrm{~d}, 21 \mathrm{~d}, 28 \mathrm{~d}$, and compared with the model group and the acupuncture group at the same time point, the results were statistically significant $(\mathrm{P}<0.05)$ (Figure 3$)$.

\section{Discussion}

\section{GV20 penetrating GB7 needling}

Head acupuncture therapy is a kind of method of treating disease by using needles to stimulate the head acupoints (or stimulation area). The effect of needling the head acupoints on treating ICH has been confirmed and recorded in ancient time. It is said in the ERON song of Compendium of Acupuncture and Moxibustion, booked in the Ming Dynasty and the most important book to study acupuncture, in which the theory of acupuncture and moxibustion is comprehensively
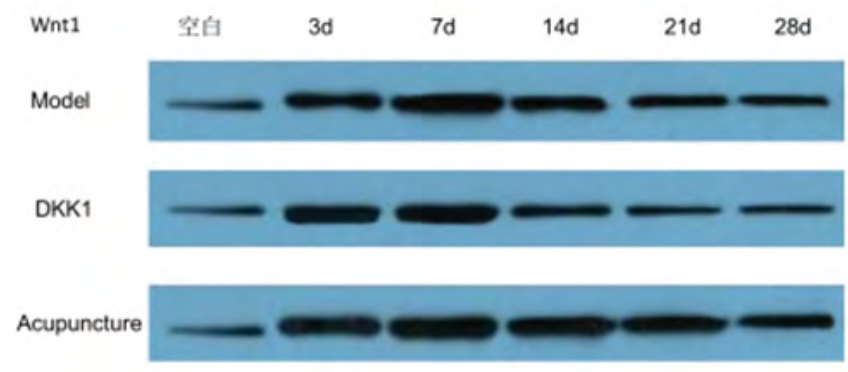

$\beta$-actin

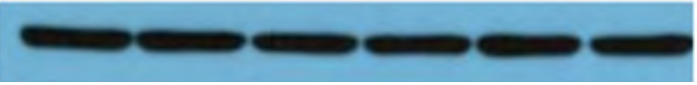

Figure 1: Effect of DKK1, acupuncture or no intervention on the protein expressionof Wnt1 in the normal brain tissues or the $\mathrm{ICH}$ brain tissues.

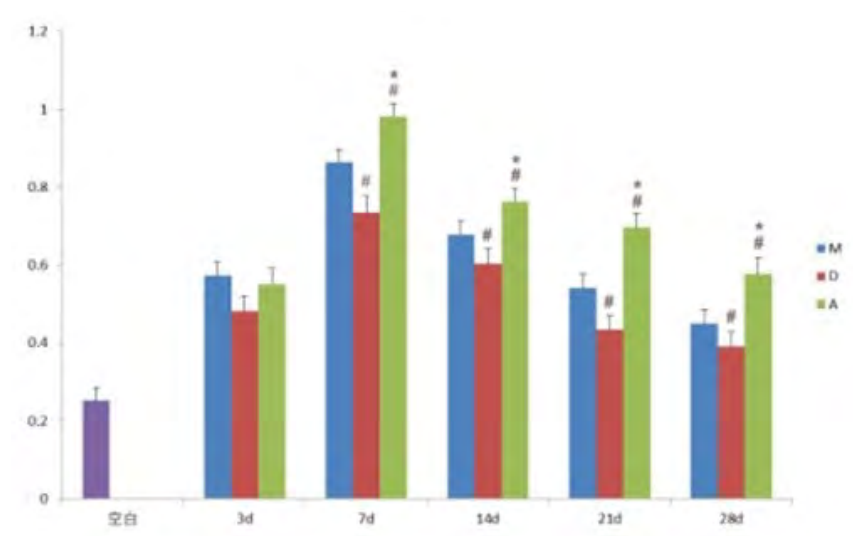

Figure 2: The protein expression of Wnt1 in the blank group, the model group the DKK1 group and the acupuncture group in $3 d, 7 d, 14 d, 21 d, 28 d$. The results are presented as the mean $\pm S D$. ${ }^{*} P<0.01$, as compared with the DKK1 group, ${ }^{*} \mathrm{P}<0.01$, as compared with the model group.

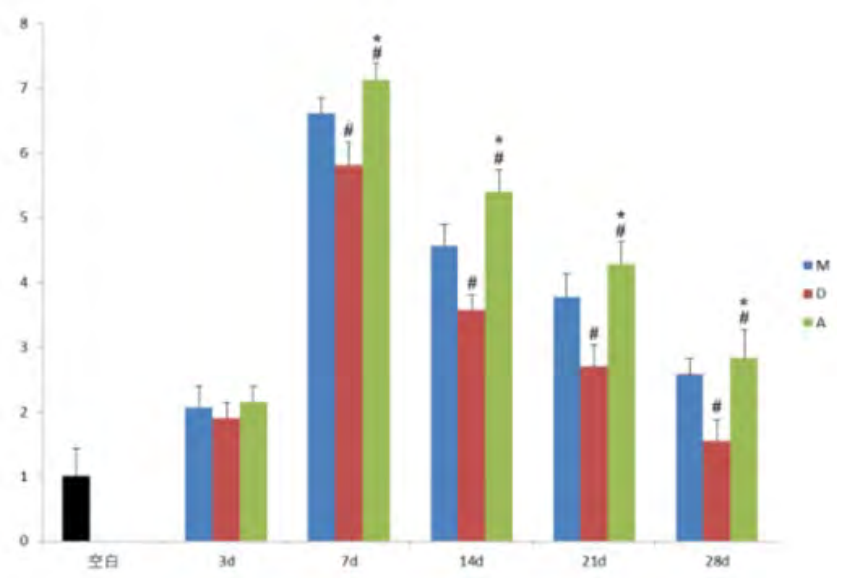

Figure 3: The mRNA expression of Wnt1 in the blank group, the model group, the DKK1 group and the acupuncture group in $3 d, 7 d, 14 d, 21 d, 28 d$. The results are presented as the mean $\pm S D$. ${ }^{*} P<0.01$, as compared with the DKK1 group, ${ }^{*} \mathrm{P}<0.01$, as compared with the model group.

discussed, that "Aphasia due to stroke is a disease most difficult to be cured. The fontanel (where GV20 is located) and GV 24 should be selected for needling. GV20 is required to be needled with more supplementation and less draining method, and the former first and the latter second. So treated, the patients can be recovered and safe." From the electrophysiology perspective, head is the volume conductor, and needling head acupoints can transmit the bioelectrical effect produced by the acupuncture stimulation to the cerebral cortex by the volume conductor, hence the excitability of neurons in the cerebral cortex is changed. This can reverse the brain neurons excitability inhibited by hemorrhagic stimulation or hematoma compression, awaken the shock or dormancy brain nerve cells, recover the excitability of neural cells in cerebral cortex, and enhance cerebral compensatory function. Therefore, the brain function improves.

GV20 penetrating GB7 is an ideal clinical needling method for the treatment of stroke. Professor Zou Wei has conducted a great deal of research on its mechanism. The parietal lobe, frontal lobe and temporal lobe, where the motor cortex and the sensory cortex locate, are underneath the area of GV20 to GB7. These three lobes are important in processing sensory information, language, and movement coordination. GV20 penetrating GB7 with rapidly twisting manipulation may help restoring consciousness and improving the ability of language and the motor function.

\section{The effect of GV20 penetrating GB7 on the expression of Wnt 1 in brain tissue of $\mathrm{ICH}$ rats}

Wnt was first discovered as a kind of cancer gene in the early 1980s. Until now, there are 19 Wnt genes found in human genome. Among those, Wnt1 is the most important gene in the process of neural development, and has the widest expression in the body. Some experimental results have showed that endogenous NSCs of both rats and humans are activated [10-13] and the active NSCs may take part in the recovery process of nervous system after an ischemic or hypoxic injury [14-19]. Besides, some data display that nerve is enhanced in hippocampus, striatum, and cortex after the brain is injured by $\mathrm{ICH}$, and Wnt signaling pathway is discovered to have the ability of regulating hippocampal neurogenesis of adult SD rats [20]. Some Chinese scholars [21] have found that the time-dependent expression of Wnt1 in the hippocampus of ischemic injury coincides 
with the process of proliferation and differentiation of NSCs. They illustrates that Wntl plays an important regulatory role in the early process of proliferation and differentiation of NSCs. The research on NSCs of ventral midbrain by Castelo-Branco et al. has discovered that Wnt1 in ventral midbrain mainly promotes the proliferation of NSCs, and is helpful for differentiation of various neurons [22]. In the process of nerve differentiation, blockade of the Wnt signaling pathway can decrease NSCs proliferation, whereas activation of the Wnt signaling pathway may cause the opposite effect [23]. Previous experiments $[24,25]$ showed that the $\mathrm{Wnt} / \beta$-catenin signaling pathway stimulates self-renewal of NSCs, and by inhibiting gliogenesis, NSCs differentiation towards neuronal phenotype could be activated as well. In addition, down-regulation of $\mathrm{Wnt} / \beta$-catenin causes degeneration of striatal synapsis, which finally causes impaired motor behaviour [26]. Herein, the expression of Wnt1 is always accompanied by the differentiation of neural cells; when cell differentiation suspends, the expression of Wnt1 stops. This signifies that the expression of Wnt1 is closely related to the differentiation and maturation of nerve cells.

The Wnt signaling pathway controls many events during embryonic development and regulates proliferation, morphology, motility, and cell fate at the cellular level. Activation of the $\mathrm{Wnt} / \beta$-catenin pathway is characterized by 1 ) uncoupling $\beta$-catenin from degradation complex after receptor engagement by Wnt ligands, 2) $\beta$-catenin nucleus translocation, 3) $\beta$-catenin interaction with lymphoid enhancerbinding factor-1/T-cell factor-1 (LEF/TCF) transcription factors, and 4) stimulation of target genes (Figure 4) [27-29]. A study also showed that Wnt family gene mRNA expression in NSCs can be isolated from rat SVZ [30]. These results suggest that the Wnt signaling pathway involves deeply in regulating NSCs proliferation and differentiation [31,32] during brain development.

This study utilizes Western-blot and RTFQ PCR methods to observe the changes of the Wntl protein/mRNA expression in brain tissue of rats at different time points. These two methods for detecting Wnt1, from protein and gene aspects, reach the same conclusion that GV20 penetrating GB7 can promote the Wnt1 protein/mRNA expression in brain tissue of ICH rats. Although the Wntl protein/mRNA expression decreases after $14 \mathrm{~d}$, the expression in the acupuncture group is still higher than that of the other groups at the same time point. Along with the increase of acupuncture times, this needling can obviously promote Wnt1 protein/mRNA expression, and urge Wnt1 to combine

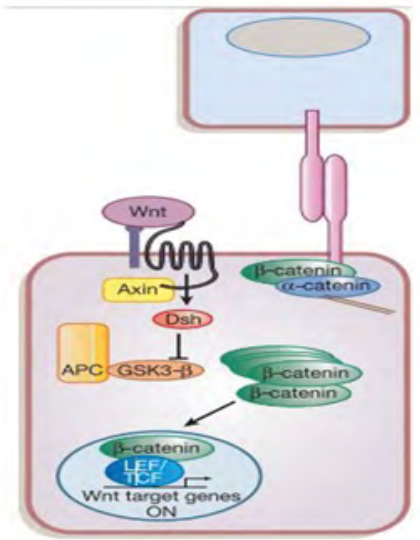

Figure 4: In the presence of Wnt signaling, $\beta$-catenin is uncoupled from the degradation complex and transloactes to the nucleus, where its binds LEF/TCF transcription factors, thus activating target genes [27]. with the receptors located on the cell membrane through autocrine and paracrine. The stimulation can further activate signaling pathway of intracellular Wnt1, and regulate the expression of downstream target genes, in order to promote the proliferation of endogenous NSCs and the differentiation of neuron. Thereby, endogenous repair mechanism of the organism itself is initiated, and the regeneration and remodeling of neural function after ICH is finally promoted.

\section{Conclusion}

This study reveals that GV20 penetrating GB7 needling method can improve the expression of the Wnt signal pathway from protein and mRNA and this result may be one of the important mechanisms of acupuncture on treating ICH.

\section{Conflict of Interest}

The authors confirm that this article content has no conflict of interest.

\section{Acknowledgements}

This study was supported by grants from NSFC (No. 81473764, No. 81273824), Natural Science Foundation of Heilongjiang Province of China (No. ZD201204) and the PhD. Programs Foundation of Ministry of Education of China (No.20102327110003).

\section{References}

1. Rao ML, Wang WZ, Huang RX (2004) Guidelines for the prevention and treatment of cerebrovascular diseases in China. Chinese Journal of Prevention of Control of Chronic Non-Communicable Diseases 2: 36.

2. Evans MJ, Kaufman MH (1981) Establishment in culture of pluripotential cells from mouse embryos. Nature 292: 154-156.

3. Liu F, Zhang GW, Zou W, Li D (2009) Nerve protection mechanism of acupuncture to the secondary injury in the rat of intracerebral hemorrhage. Chinese Journal of Primary Medicine and Pharmacy 16: 420-422.

4. Zhang GW, Zou W, Liu F (2010) Experimental Study on the Influence of Acupuncture on GDNF Expression in Rats of Acute Intracerebral Hemorrhage. Journal of Inner Mongolia University for Nationalities 2: 191-193.

5. Shen J, Xie L, Mao X, Zhou Y, Zhan R et al (2008) Neurogenesis after primary intracerebral hemorrhage in adult human brain. J Cereb Blood Flow Metab 28: 1460-1468

6. Masuda T, Isobe Y, Aihara N, Furuyama F, Misumi S, et al. (2007) Increase in neurogenesis and neuroblast migration after a small intracerebral hemorrhage in rats. Neurosci Lett 425: 114-119.

7. Zou W, Li D, Yu XP, Dai XH, Teng W, et al. (2009) Clinical study on acupuncture treatment of acute hypertensive intracerebral hemorrhage. Information on Traditional Chinese Medicine 5: 104-105.

8. Liu B, Zou Wei, Tang Q, Yan PY (2014) Effects of Cluster Scalp Acupuncture Combined with Enriched Environment Stimulation on Proliferation and Migration of Endogenous Neural Stem Cells in Neonatal Rats with Hypoxicischemic Brain Damage. Chin J Rehabil Theory Pract 20: 533-536.

9. Berdson JB (1986) Rat middle cerebral artery occlusion: evaluation of the model and development of a neurologic examination. Stroke 17: 472-473.

10. Santilli G, Lamorte G, Carlessi L, Ferrari D, Rota NL, et al. (2010) Mild hypoxia enhances proliferation and multipotency of human neural stem cells. PLoS One 5: 8575.

11. Jin K, Wang X, Xie L, Mao XO, Zhu W, et al. (2006) Evidence for strokeinduced neurogenesis in the human brain. Proc Natl Acad Sci USA 103: 13198-13202.

12. Zhang $\mathrm{P}$, Liu $\mathrm{Y}$, Li J, Kang Q, Tian $\mathrm{Y}$, et al. (2006) Cell proliferation in ependymal/subventricular zone and nNOS expression following focal cerebral ischemia in adult rats. Neurol Res 28: 91-96.

13. Zhang P, Liu Y, Li J, Kang Q, Tian Y, et al. (2007) Decreased neuronal nitric oxide synthase expression and cell migration in the peri-infarction after focal cerebral ischemia in rats. Neuropathology 27: 347-354.

14. Hou SW, Wang YQ, Xu M, Shen DH, Wang JJ, et al. (2008) Functional 
Citation: Wu W, Dai X, Zou W, Yu X, Teng W, et al. (2017) GV20 Penetrating GB7 on the Expression of Wnt1 in Brain Tissue of Rats with Intracerebral Hemorrhage. Med Chem (Los Angeles) 7: 388-392. doi: 10.4172/2161-0444.10485

integration of newly generated neurons into striatum after cerebral ischemia in the adult rat brain. Stroke 39: 2837-2844.

15. Miles DK, Kernie SG (2008) Hypoxic-ischemic brain injury activates early hippocampal stem/progenitor cells to replace vulnerable neuroblasts. Hippocampus 18: 793-806.

16. Nakayama D, Matsuyama $T$, Ishibashi-Ueda $H$, Nakagomi $T$, Kasahara $Y$, et al. (2010) Injury-induced neural stem/progenitor cells in post-stroke human cerebral cortex. Eur J Neurosci 31: 90-98.

17. Ohira K, Furuta T, Hioki H, Nakamura KC, Kuramoto E, et al. (2010) Ischemiainduced neurogenesis of neocortical layer 1 progenitor cells. Eur J Neurosci 13: $173-179$.

18. Shen CC, Yang YC, Chiao MT, Cheng WY, Tsuei YS, et al. (2010) Characterization of endogenous neural progenitor cells after experimental ischemic stroke. Curr Neurovasc Res 7: 6-14.

19. Wang XL, Zhao YS, Hu MY, Sun YQ, Chen YX, et al. (2013) Umbilical cord blood cells regulate endogenous neural stem cell proliferation via hedgehog signaling in hypoxic ischemic neonatal rats. Brain Res 1518: 26-35.

20. Ohira K (2011) Injury-induced neurogenesis in the mammalian forebrain. Cell Mol Life Sci 68: 1645-1656.

21. Xing XS, Lv WL (2007) Role of Wnt-1 in the proliferation and differentiation of hippocampus endogenous neural stem cells after cerebral ischemia reperfusion in rats. Journal of Clinical Rehabilitative Tissue Engineering Research 3: 412-414.

22. Castelo-Branco G, Sousa KM, Bryja V, Pinto L, Wagner J, et al. (2006) Ventral midbrain glia express region-specific transcription factors and regulate dopaminergic neurogenesis through Wnt-5a secretion. Mol Cell Neurosci 31: 251-262.

23. Qi C, Zhang J, Chen X, Wan J, Wang J, et al. (2017) Hypoxia stimulates neural stem cell proliferation by increasing HIF? $1 \alpha$ expression and activating Wnt/ $\beta$ catenin signaling. Cell Mol Biol 63: 12-19.

24. Kalani MY, Cheshier SH, Cord BJ, Bababeygy SR, Vogel H, et al. (2008) Wntmediated self-renewal of neural stem/progenitor cells. Proc Natl Acad Sci USA 105: 16970-16975.

25. Kunke D, Bryja V, Mygland L, Arenas E, Krauss S (2009) Inhibition of canonical Wnt signaling promotes gliogenesis in P0- NSCs. Biochem Biophys Res Commun 386: 628-633.

26. Galli S, Lopes DM, Ammari R, Kopra J, Millar SE, et al. (2014) Deficient Wnt signaling triggers striatal synaptic degeneration and impaired motor behaviour in adult mice. Nat Commun 5: 4992.

27. Reya T, Clevers $\mathrm{H}$ (2005) Wnt signaling in stem cells and cancer. Nature 434 843-850.

28. van de Wetering M, Cavallo R, Dooijes D, van Beest M, van Es J, et al. (1997) Armadillo coactivates transcription driven by the product of the Drosophila segment polarity gene dTCF. Cell 88: 789-799.

29. Willert K, Nusse R (1998) Beta-catenin: a key mediator of Wnt signaling. Curr Opin Genet Dev 8: 95-102.

30. Morris DC, Zhang ZG, Wang Y, Zhang RL, Gregg S, et al. (2007) Wnt expression in the adult rat subventricular zone after stroke. Neurosci Lett 418 170-174.

31. Hirabayashi $\mathrm{Y}$, Itoh $\mathrm{Y}$, Tabata $\mathrm{H}$, Nakajima K, Akiyama T, et al. (2004) The wnt/beta-catenin pathway directs neuronal differentiation of cortical neural precursor cells. Development 131: 2791-2801.

32. Poschl J, Grammel D, Dorostkar MM, Kretzschmar HA, Schuller U (2013) Constitutive activation of beta-catenin in neural progenitors results in disrupted proliferation and migration of neurons within the central nervous system. Dev Biol 374: 319-332. 